\title{
De la régénération à la dégénérescence : \\ La science de l'homme face à 1848.
}

«Sur quoi peut reposer l'égalité des droits ? Sur l'égalité des aptitudes, c'est-à-dire, sur l'égalité des âmes, l'égalité de la raison des hommes proclamée par Descartes lui-même. L'intelligence est cette âme, cette raison, manifestée par des organes plus ou moins parfaits »

François Eusèbe de Salles, Histoire générale des races humaines ou philosophie ethnographique, Paris, Benjamin Duprat, 1849, p. 298.

La révolution de 1848 a t-elle eu une influence sur les discours scientifiques sur l'homme ? Et ces derniers ont-il inversement joué un rôle dans l’interprétation des événements de 1848 ? Questions ouvertes et difficiles, pour deux raisons au moins. Il semble impossible d'abord de dégager une représentation claire, partagée et consensuelle sur ce qu'est l’Homme sous la Monarchie censitaire ou le second Empire. Même si l’on écarte ici les processus de définitions des genres (masculin/féminin), plusieurs anthropologies sont en conflit ouvert. Il serait ensuite téméraire d'avancer une réponse nette à la lumière d'une histoire des «sciences humaines » qui reste encore largement lacunaire pour la première moitié du XIXe siècle. Plutôt que d'inventorier les théories concurrentes et les polémiques qu'elles alimentent (matérialisme/spiritualisme, physiologie/psychologie, monogénisme/polygénisme, etc.), cette contribution évoquera la trajectoire de la phrénologie et l'échec de son projet social, que scelle la révolution de 1848. Le contraste entre cette théorie et le succès au début des années 1850 de l' « anthropologie morbide » de Morel permettra d'avancer ensuite quelques hypothèses.

\section{La phrénologie, nouvelle science de l'homme.}

L’anthropologie du XVIIIe siècle peut être caractérisée, si l'on néglige des variations souvent importantes entre les auteurs, par le projet exposé par les Cornélius de Pauw, J-F Blumenbach, G. Forster et autres P. Camper. Il s'agit de produire une nouvelle connaissance sur l'homme, en tant qu'espèce animale. Ce genre d' « histoire naturelle de l'homme » persiste jusqu'au milieu du XIXe siècle, non sans subir un profond remaniement car si l'humanisme des Lumières n'est pas le temps d’une adhésion générale à l’image du «bon sauvage », il se caractérise par la 
croyance partagée de l'universalisme du genre humain ${ }^{1}$. Quelle que soit l'échelle des êtres et des peuples proposée par les auteurs, on s'accorde alors à penser que tous les hommes participent de la même espèce, de la même humanité. Nulle chute individuelle ou collective ne semblait inéluctable, et le terme de « régénération » se retrouvent fréquemment dans les libelles, brochures et les pamphlets anonymes qui précédèrent 1789. De simple substantif, la « régénération » devient rapidement un "programme tout à la fois politique, philosophique, physique et moral » qui connaîtra de multiples déclinaisons au XIXe siècle ${ }^{2}$. Par contraste avec les Lumières, l'ethnologie qui s'impose peu à peu dans la première moitié du XIXe siècle se construit sur la dissolution du concept d'homme universel, auquel elle substitue celui de races ${ }^{3}$. Différentialiste et classificatoire, elle cherche à hiérarchiser les peuples en fonction de leurs aptitudes mentales et physiques: l'égalité n’est plus alors qu'une chimère philosophique démentie à la fois par la littérature contre-révolutionnaire et une frange de physiologistes positivistes. La doctrine des races devient l'une des clefs de lecture de l'histoire de France des Guizot et des frères Thierry, et la physiologie tend à s'appliquer à l'histoire entière de l’humanité, semblant même confirmer l'histoire biblique chez le très catholique abbé Frère.

C’est dans ce contexte très brièvement décrit que la phrénologie est introduite en France sous l'Empire ${ }^{4}$. S'inscrivant dans le cadre d'une histoire naturelle de l'homme, son objectif apparaît de prime abord en continuité avec le projet de l’Idéologie : il s’agit de donner « une direction sûre à l'éducation » et une «impulsion convenable aux différentes passions, pour en faire les instruments du bonheur particulier de l'individu et du bien général de la société » ${ }^{5}$. Pour y parvenir, il faut établir ce que le docteur François-Joseph Gall (1758-1828) appelle explicitement une «véritable science de l'homme ${ }^{6}$. Le médecin viennois veut forger cette nouvelle science avec une représentation novatrice du fonctionnement cérébral, qui peut être caractérisée en six propositions. La première pose que les penchants, les qualités morales et les facultés intellectuelles de l'homme sont, comme chez les animaux, innés. La seconde relie l'exercice de ces facultés à l'organisation physique de l'individu. La troisième affirme que le cerveau est l'organe de tous les penchants, de toutes les qualités et de toutes les facultés. La

\footnotetext{
${ }^{1}$. Sur les vicissitudes du bon sauvage au XVIIIe siècle, voir Michèle Duchet, Anthropologie et histoire au siècle des Lumières, Paris, Albin Michel, 1995 (1971), postface de Claude Blanckaert.

2. M. Ozouf, L’homme régénéré. Essais sur la Révolution française, Paris, Gallimard, 1989, p. 128. Sur la réaction polémique à ce mouvement, voir le recueil collectif, Régénération et reconstruction sociale entre 1780 et 1848, Paris, Vrin, 1978.

3 . Claude Blanckaert, "Le système des races » in Isabelle Poutrin (Ed.), Le XIXe siècle. Science, politique et tradition, Paris, Berger-Levrault, 1995, pp. 21-41 ; «On the origins of French ethnology (William Edwards and the Doctrine of Race)» in George W. Stocking (Ed), Bones, Bodies, Behavior, The University of Wisconsin Press, 1988 (a), pp. 18-51.

${ }^{4}$. Sur la phrénologie française, voir Georges Lantéri-Laura, Histoire de la phrénologie, Paris, PUF, 1993 (1970).

5 . François-Joseph Gall, Discours d'ouverture lu par M. le Docteur Gall à la première séance de son cours public sur la physiologie du cerveau, le 15 janvier 1808, Paris, Firmin Didot, 1808, pp. 8-9.

. Ibid., p. 6.
} 
quatrième, que le cerveau n'est pas un organe homogène, et qu'il est décomposable en autant d'organes particuliers qu'il existe de facultés (27 selon Gall). La cinquième proposition affirme que le développement d'une faculté est proportionnel au volume de son organe : les différentes facultés agissent donc en synergie en fonction de leur importance respective. La dernière proposition est à l'origine de toutes les autres : la forme du crâne correspondant pour l'essentiel à l'enveloppe extérieure du cerveau, on peut évaluer le développement relatif de chaque faculté en tâtant les saillies et les méplats de la boîte crânienne.

Face au déclin sous l’Empire de l'Idéologie, le monisme de la physiologie phrénologique joue une fonction de relais auprès des médecins qui souhaitent poursuivre le programme de recherche lancé par Cabanis. Gall présente en effet sa théorie cérébrale à un moment où les études «psychologiques» (au sens actuel du terme) sont l'objet d'une polémique entre les tenants d'une perspective purement philosophique et ceux qui prônent une approche plus physiologique. Le débat de méthode initié par les Idéologues fait alors de la psychologie un enjeu de savoir, de compétences et d'institutions. L'esprit, la conscience ou l'âme étaient chez les spiritualistes la condition nécessaire pour que l'individu puisse atteindre sa dignité de sujet. Sans conscience, le sujet n’était plus. Comme le rappelle l'exergue du polygraphe Eusèbe de Salles, on restait ici au sein d'un cadre fixé par la représentation du cogito cartésien. L’âpreté des polémiques était liée au déplacement opéré par les médecins en général et les phrénologistes en particulier, qui posait le sujet comme objet de savoir dans une égale mise à distance de l’âme et de la conscience.

Malgré la levée de boucliers qu’elle provoque, la doctrine phrénologique parvient à convaincre sous la Restauration un nombre croissant de médecins de sensibilité bonapartiste et libérale. Certains phrénologistes s’engagent dans la Charbonnerie sous la Restauration (B. Appert, M.-A. Dupoty, G. Ferrus, G. Fossati, etc.) et Benjamin Appert contribue à diffuser la théorie de Gall dans l’opposition libérale. Une Société phrénologique de Paris est créée début 1831 dans le but « de propager et de perfectionner la doctrine de Gall ». Sa devise, «Au progrès des Lumières ", tranche à la fois avec la philosophie des Congrégations catholiques de la Restauration et l'éclectisme alors triomphant de Victor Cousin, trop timoré aux yeux de nombreux médecins. Loin d’être une simple théorie, la phrénologie devient un programme de recherche doublé d’un phénomène de société. Exception faite peut-être du magnétisme, on n’a jamais vu en effet dans cette première moitié du XIXe siècle une société savante se créer pour la défense d’une théorie spécifique. Ce fait montre que la doctrine de Gall a acquis, pour une petite vingtaine d'années, un statut quasiment comparable dans le savoir médical à la méthode anatomo-clinique. Véritable 
programme de recherche donc, ou tendant à le faire croire, elle se distingue toutefois de l'anatomo-clinique en ce qu'elle se double d'une résonance sociale, liée aux applications qu’elle revendique. Forte de 200 adhérents, la Société phrénologique est, à la différence de l'anatomopathologie, politisée. Deux générations de libéraux s’y retrouvent: le comte de Lasteyrie et le baron Ternaux apportent la caution de leur âge, tandis qu'on relève la présence du jeune duc de Montebello, Léon Faucher, le nouveau médecin-maire du 4ème arrondissement Félix Cadet de Gassicourt, son adjoint Viguier, le député Emmanuel de Las Cases (fils), le baron Noël Girard et le député Auguste Saint-Aignan. Au delà de son objectif statutaire donc, la société phrénologique visait un but plus large : "Sortis de cette admirable révolution [1830] et forts de notre origine, explique Casimir Broussais, nous voulons cultiver avec toute l'ardeur qu'elle réclame, cette science qui doit fournir aux législateurs, aux instituteurs, aux moralistes, de si précieuses données pour obtenir, dans la législation, l'éducation et la philosophie ces perfectionnements demandés aujourd'hui avec tant d'unanimité » ${ }^{7}$.

\section{Changer l'homme et la société}

L’engouement pour la phrénologie est tel dans ces premières années de la Monarchie de Juillet qu'une seconde institution privée voit le jour en 1832 sous le nom de Société anthropologique de Paris. Cette société savante française est ainsi la première à intégrer le terme d'anthropologie dans sa désignation, plus d'un demi-siècle avant que Broca ne fonde la sienne. Son but est cette fois encore de peser sur l'organisation sociale : «La société a pour but de connaître la nature de l'homme, de propager cette connaissance et d'indiquer les applications salutaires qui peuvent en être faites aux institutions sociales ${ }^{8}$. Ses membres actifs sont Spurzheim et William Edwards (alors membre de l'Académie des sciences morales et politiques). On relève, parmi ses adhérents, des membres de la Société phrénologique de Paris comme Pierre Foissac (secrétaire), David d'Angers, Las Cases, Roberton, le comte de Lasteyrie, Las Cases, David Richard et le nom, moins attendu, de Louis de Potter. La Société anthropologique n’a probablement pas dépassé le cap d’une année d'existence mais ses statuts indiquent qu'elle était beaucoup moins intéressée par la médecine que par les applications politiques de la phrénologie. En rester à l'approche déterministe de Gall, c’était se couper de toutes les revendications que portait le discours réformateur de J.-G. Spurzheim (premier disciple dissident du maître) et - à terme - des

\footnotetext{
7. C. Broussais, « Compte-rendu des travaux de la séance annuelle », 1832, Journal de la Société phrénologique de Paris, vol. I, $\mathrm{n}^{\circ} 1, \mathrm{p} 82$.

8. Pierre Foissac, «La tête de Bichat devant la Société anthropologique; et les localisations cérébrales », L'Union médicale, $1877, n^{\circ} 145$, pp. 869-873.
} 
attentes politiques du groupe social qui semblait le plus réceptif à la nouvelle théorie. Le propos tenu par le docteur Lacorbière lors du congrès de l'Institut historique de 1835, montre que l'on était prêt à composer avec l’esprit du maître, pour renouer avec l'élan réformiste des Lumières : «Si nous convenons avec Gall que la perfectibilité morale de l'espèce humaine est confinée dans les limites de son organisation, que ces limites ont été posées invariables par le Créateur, il n'en est pas moins vrai qu'il est impossible de fixer un terme aux connaissances et aux découvertes, et qu'il reste immensément à faire à l'homme pour atteindre les bornes de sa puissance, le summum de sa perfection! » ${ }^{9}$. Gall proposait d'adapter la société, Spurzheim affirmait qu'elle devait changer, parce qu'elle était «contre nature ». C’est du moins ainsi que les phrénologistes français interprètent leur science, s’intéressant moins au fil du temps à faire de nouvelles découvertes en physiologie qu’à exiger une réforme de la société. Fossati explique que la «science de l'éducation » recevra une «lumineuse direction » de la phrénologie car elle démontre la nécessité d'adapter l'enseignement aux dispositions natives des enfants. On reprenait quasiment, mot pour mot, le vieux rêve de Cabanis : «Après avoir passé des siècles ne s'occupant que du perfectionnement de la race des chevaux, des moutons et des chiens, écrivait Fossati, on s'occupera peut-être un peu du perfectionnement de la race humaine ${ }^{10}{ }^{. . .}$

Ce caractère appliqué de la doctrine est très net dans le champ de la criminalité ${ }^{11}$. Si le réformisme des phrénologistes reprend à la lettre la volonté de repenser l'économie des peines de Gall, il en modifie considérablement l'esprit, qui s'inspire de Spurzheim et de l'optimisme thérapeutique des aliénistes de cette première moitié de siècle ${ }^{12}$. Les phrénologistes ne s’arrogent plus seulement la mission humanitaire de soustraire les aliénés-criminels à la peine de mort mais ils réclament une analyse scientifique, un diagnostic et un droit de traitement de tous les condamnés, qui doivent être classés en fonction de leurs capacités naturelles. Pour Félix Voisin, les têtes de criminels sont «comme les grandes têtes morales et intellectuelles placées par la nature en dehors de l'espèce humaine [...] le front est étroit, déprimé, fuyant en arrière, bas, nоueux, irrégulier, et la partie supérieure de leur tête est évidée comme le toit d'un couvreur ${ }^{13}$. Mais le phrénologiste est loin de penser que cette marque d'exclusion est

\footnotetext{
9. Beunaiche de la Corbière, Réponse aux objections faites à la phrénologie, Paris, Pihan Delaforest, 1836, p. 28.

10. Giovanni Fossati, Questions philosophiques, sociales et politiques traitées d'après les principes de la physiologie du cerveau, Paris, Librairie d'Amyot, 1869, p. 75. A comparer avec P.-J.-G. Cabanis, Rapports du physique et du moral de l'homme, Paris-Genève, Ressources, 1980 (édition de 1844), pp. 299-300.

11. Sur cet aspect de la phrénologie, M. Renneville, La médecine du crime, Villeneuve d'Ascq, Presses universitaires du Septentrion, 1997, t. I, pp. 252-520.

12. M. Gauchet et G. Swain, La pratique de l'esprit humain, Paris, Gallimard, 1980, pp. 41-51 ; G. J. Makari, « Educated insane : a nineteenth-century psychiatric paradigm », Journal of the History of Behavioral Sciences, 1993, vol. 29, n 1, pp. 8-21.

${ }^{13}$. F. Voisin, « De l'organisation cérébrale défectueuse de la plupart des criminels », Bulletin de l'académie de médecine, 1837, p. 911.
} 
irrémissible. La laideur ou l'infirmité physique sont liées ici - dans la continuité des révolutionnaires comme Barère, Lanthenas ou Le Peletier - à la condition sociale. Rappelonsnous cette leçon que Camille reçoit dans l'Icarie rêvée par Cabet: «Tu sais combien l'abondance ou le besoin, le calme ou les angoisses, le bonheur ou l'indigence, influent sur la beauté physique et sur l'intelligence! Tu sais combien les enfants des riches sont généralement plus beaux que ceux des pauvres, combien certaines populations sont belles, et combien certaines autres sont abâtardies par la misère ! » ${ }^{14}$. Les phrénologistes le savent effectivement, et leurs monstres sont plus proches du panthéon hugolien que de la tératogenèse d'Isidore Geoffroy Saint-Hilaire. La naturalisation de la déviance ne cautionne pas ici de mesures répressives. Affirmer que les idiots ou les criminels sont placés par la nature « en dehors de l'espèce humaine » est une incitation à prendre en charge ces exclus. Il n'était pas encore temps de mettre en avant l'éradication des incorrigibles ou des pervers par nature. Félix Voisin mettra d'ailleurs en pratique ses idées rééducatives en ouvrant à Issy-les-Moulineaux un éphémère Institut « orthophrénique ».

\section{L'échec d'un projet}

Cet engagement des phrénologistes pour un système éducatif alternatif, basé sur la présélection des potentialités de chacun, ne fut pas l'élément le moins important dans le débat avec les antiphrénologistes. Le fait qu'un Félix Voisin s'intéresse à des catégories d'individus jusqu'ici délaissés (les enfants «idiots ») est ignoré par Népomucène Lemercier, qui dénonce les dangers du nouvel établissement de l'aliéniste le 23 février 1835 à l’Académie des sciences : «une véritable institution orthophrénique ne pourrait [être dirigée] que par une créature surhumaine ou, s'il en existait de connu, par un demi-Dieu. Eh! Quel homme assez superbe aurait l'orgueil de s'annoncer comme le directeur infaillible des agents de l'esprit et des passions animées qui l'élèvent ou qui l'abattent ? ». Aussi bien selon Lemercier, si l'orthopédie de Voisin était une véritable science, la phrénologie ne vaudrait pas plus que l'astrologie car ses prétentions à classer et éduquer les hommes constituaient une renonciation au libre-arbitre et à la liberté de conscience. Lemercier classait les « cranologues » entre le catholicisme fanatique et l'utopie des Saint-Simoniens ce qui n’était pas si mal vu : «O révérends cranologues, bientôt successeurs des médecins de l'âme, déclamait-il, si vos pratiques mortifiantes se joignent à votre enseignement, je n'hésite pas à affirmer qu'en comparant les doctrines théocratiques et les vôtres, toutes idéales, toutes fantastiques, la prudence du siècle dernier les eût ironiquement

\footnotetext{
${ }^{14}$. E. Cabet, Voyage en Icarie, Paris-Genève, Slatkine, 1979 (1842), p. 121.
} 
assimilées à ces vaporeuses hallucinations de la secte Saint-Simonienne prédisposant ses illuminés des deux sexes à se classer en proportion de leurs capacités. Eh ! par qui seront-elles et pourront-elles être évaluées ? par les présomptions des pères catéchisant, tous enclins à se considérer chacun comme étant les premiers flambeaux de leur mystique et licencieuse communauté » 15 .

La polémique va prendre en France des allures de croisade lorsque le docteur Laurent Cerise (1807-1869), fidèle disciple de Buchez et de l'abbé Badiche, dédie en 1835 sa réfutation aux élèves de l'école de médecine de Paris : «Cet examen critique du système phrénologique vous est adressé par nous qui avons décidé de combattre, avec énergie et persévérance, pour la sainte cause de l'unité et de la fraternité chrétienne ${ }^{16}$. Rejetant clairement l'inductivisme prôné par les phrénologistes, Cerise subordonnait explicitement la « véracité » d’une théorie à sa «moralité » ${ }^{17}$. L’expansion de la phrénologie fut également contrecarrée par la puissance retrouvée de la Compagnie de Jésus. Louis-Ignace Moreau par exemple, dont les études sont publiées sous les auspices de Monseigneur de Luca dans les Annales des Sciences religieuses, dénonce haut et fort le matérialisme phrénologique qui «proclame au dedans de l'homme le règne de la matière »: c'est un «paganisme interne " ${ }^{18}$. La plupart des arguments des catholiques relevaient de la théologie. Mais comment s’en étonner, lorsque Buchez défendait, depuis ses articles dans L'Européen jusqu'à son cours d'introduction aux sciences médicales «l'universalité du critérium moral »? C’est d'ailleurs au nom de la morale, comme Cerise en 1836, que Buchez écartait la science phrénologique en éludant la discussion de ses arguments physiologiques $^{19}$. Retenons l'argument, qui fonde ici un refus, et légitimera bientôt un engagement pour la théorie des dégénérescences : «Il vous sera dit dans le cours de vos études, et c'est la doctrine phrénologique qui se chargera de vous l'enseigner, que tous les actes des hommes sont les conséquences nécessaires, ou les résultats nécessaires, des aptitudes déposées en eux : que ces aptitudes elles-mêmes résultent de l'organisation même du cerveau ; et que, par conséquent, la disposition organique de l'appareil cérébral prédétermine nécessairement tous les actes humains. A cette argumentation vous répondrez : que toute doctrine semblable conclut à la non-existence de l'âme, puisque, tous les actes humains étant déterminés par la seule

\footnotetext{
${ }^{15}$. N. Lemercier, Réflexions sur le danger des applications de la conjecturale doctrine orthophrénique, Paris, Firmin Didot frères, 1835 , pp. 20-21.

16. L. Cerise, Exposé et examen critique du système phrénologique, considéré dans ses principes, dans sa méthode, dans sa théorie et dans ses conséquences, Paris, Trinquart, 1836. Cerise est un proche ami de Buchez et il collabore à L'Européen, fondé en 1835 pour diffuser les opinions morales et philosophiques du maître.

17. L. Cerise, ibid, p XI.

${ }^{18}$. L.-I. Moreau, Du matérialisme phrénologique, Paris Debécourt, 1843, pp. 40-41.

${ }^{19}$. Elizabeth. A. Williams affirme que Buchez laissa le soin de cette réfutation à Cerise. Cf. The physical and the moral. Anthropology, physiology and philosophical medicine in France. 1750-1850, Cambridge, CUP, 1994, p. 221
} 
organisation de l'appareil cérébral, l'âme existe comme si elle n'existait pas. Or, toute doctrine qui conclut à la négation de l'une des existences que la morale suppose et démontre, étant par cela seul démontrée fausse, vous serez par cela seul dispensés de toute autre vérification. D’ailleurs, cette doctrine ne nie-t-elle pas le premier fondement de toute morale? - la libre responsabilité des hommes $\gg{ }^{20}$.

François Broussais a beau siéger à l'Académie des sciences morales et politique, restaurée en 1832, il ne parviendra pas à faire contrepoids devant les arguments moralisateurs, la philosophie éclectique emmenée par Victor Cousin et l'anthropologie officielle de l'Académie ${ }^{21}$. Le cap de 1840 est marqué d’une série d'événements qui annonce son déclin à venir. Il y a d’abord un phénomène de génération, marqué par la disparition de phrénologistes aussi importants que Bailly de Blois (qui décède en 1837), François Broussais (1839) et le contre-amiral Jules Dumont d’Urville, qui décède dans l'accident de chemin de fer du 8 mai 1842. L'amorce du déclin de la phrénologie est perceptible ensuite dans le champ des études anthropologiques. L’échec de la Société anthropologique de Paris en 1832 et la fondation de la Société ethnologique de Paris en 1839 en sont les signes institutionnels les plus évidents. Malgré la participation et le patronage intellectuel de William Edwards, qui en est le premier président, cette société se garde de toute référence à la phrénologie. Parallèlement, un grand nombre de membres de la Société phrénologique adoptent une attitude très critique vis-à-vis de la monarchie censitaire. Beaucoup pensent dès les fin des années trente que la révolution reste à faire. La Société phrénologique de Paris perd aussi à fin de l’année 1841 un de ses pivots avec l’écrivain socialiste Auguste Luchet, qui part en exil. Dans le domaine scientifique, les critiques élèvent la voix au début de ces années 1840 ; avec Lélut, membre de l’Académie des sciences morales et politiques, Flourens, secrétaire perpétuel de l’Académie des sciences et Dubois d’Amiens, membre de l'Académie de médecine. Enfin, la phrénologie est de plus en plus souvent compromise dans des applications que Gall avait condamnées et le brouet des épigones littéraires la discrédite peu à peu dans la communauté médicale. Ces accrus se développent au détriment du programme initial. Les successeurs de Gall, victimes de leur succès, tentent vainement de se démarquer de la crânomancie populaire ${ }^{22}$. Rien n’y fait et il n’y a plus

\footnotetext{
20. P. Buchez, Introduction à l'étude des sciences médicales. (Leçons orales recueillies et rédigées par Henry Belfield Lefevre), Paris, Eveillard et Cie, 1838, p. 155.

${ }^{21}$. F. Broussais donnera deux importants mémoires «Mémoire sur l'association du physique et du moral ", Mémoires de l'Académie des sciences morales et politiques, 1837, vol. I, pp. 113-199 et « Du sentiment de l'individualité, du sentiment personnel et du moi ", ibid., 1841, vol. III, pp. 91-146. Sur l'opposition Broussais/Cousin à l'ASMP, voir Sophie-Anne Leterrier, L'Institution des sciences morales (17895-1850), Paris, L’Harmattan, 1995, pp. 185-193.

${ }^{22}$. F. Tellier, « De l'exploitation de la phrénologie », La phrénologie, Paris, 1837, pp. 3-4.
} 
désormais une phrénologie, mais plusieurs : celle des médecins, celle des artistes et celle des vulgarisateurs, qui fait florès sous la Monarchie de juillet.

\section{La science de l'homme en révolution}

1848 sonne le glas de la phrénologie scientifique. Tout commence bien pourtant. Dans l'attente de l'élection de l'assemblée constituante, Fossati fait le 3 avril 1848 au Palais-Royal une nouvelle lecture de sa conférence sur le choix phrénologique d’un bon législateur devant une assemblée composée de membres de la Société phrénologique et de quelques professeurs et anciens sociétaires de l’Athénée. Cette réunion aboutit à la création d’un club qui prend le nom de Club des droits civiques. Fossati est déclaré président par acclamation ${ }^{23}$. Il organise pour le 18 avril suivant la dernière séance de la Société phrénologique en présence des membres du Club des droits civiques et de personnes invitées. Fossati reprend pour l'occasion un discours de 1841 sur l'urgence de satisfaire le besoin moral des peuples... La Société phrénologique croit réaliser enfin ce qu'elle avait toujours voulu être: un guide pour l'action politique et l’organisation sociale. Ses partisans se mobilisent. Félix Voisin adresse aux membres de l'Assemblée constituante un mémoire pour l'abolition de la peine de mort, Stanislas Tanchou propose une réforme du système hospitalier par l'instauration de «nosocom ", sorte de dispensaires ouverts au peuple ; et Pierre Béraud publie un ouvrage de vulgarisation de phrénologie «appliquée à la philosophie, aux moeurs et au socialisme » qui ne tient pas les promesses du titre ${ }^{24}$. Peu de choses finalement, et encore moins de résultats, en regard des ambitions affichés dans la décennie précédente. Malgré quelques ouvrages de synthèse, la greffe avec les systèmes saint-simoniens et fouriéristes n’a pas pris. En fait, la phrénologie est déjà moribonde lorsque la révolution éclate. Effectuée sous la pression des événements, l'ultime transformation de ses statuts marque sa disparition car il n’y aura plus - à notre connaissance - de réunions officielles de la Société après 1848.

1848 semble dans un premier temps effacer les divisions des médecins au profit d'un engagement dans la politique. Si ces derniers sont beaucoup moins représentés à l'Assemblée constituante que les avocats, du moins sont-ils à sa tête avec Buchez et les docteurs Bixio, Recurt et Trélat (vice-présidents). Au-delà de cette reconnaissance politique, c’est tout le corps

\footnotetext{
${ }^{23}$. Selon l'aveu de Fossati, ce club politique n'a pas duré longtemps. Fossati, op. cit., 1869, p. 151.

${ }^{24}$. F. Voisin, Mémoire en faveur de l'abolition de la peine de mort, adressé aux représentants du peuple, Paris, Garnier Frères, 1848 ; S Tanchou, Sur les hôpitaux, Paris, Germer Baillière, 1848 ; P. Béraud, De la phrénologie humaine, appliquée à la philosophie, aux moeurs et au socialisme, Paris, Durand, 1848.
} 
médical qui vibre pour cette révolution ${ }^{25}$. Jules Guérin, qui avait pourfendu les disciples de Gall dans sa Gazette médicale, tient dès le début du mois de mars à « saluer la révolution ». "Partout des médecins éclairés ont été appelés à prendre part à l'oeuvre de régénération politique et administrative, constate t-il [...] On commence à comprendre le caractère général et élevé de la médecine ». Et Guérin d’ajouter, dithyrambique : «Il est bien vrai que la profession médicale est d'essence libérale et républicaine, qu'elle façonne l'esprit à la haine du préjugé et à l'amour du progrès. Mais ce qui commence aussi à s'apercevoir, c'est que la médecine embrasse le faisceau des véritables sciences politiques et gouvernementales; qu'elle touche par tous les points à l'économie politique; qu'elle peut mieux que toute autre aider à l'amélioration des classes pauvres, des classes ouvrières et de l'espèce humaine entière; que l'éducation publique, les réformes législatives, ne sauraient être mieux renseignées que par elle, tant par les notions de psychologie positive qu'elle peut fournir, que par une appréciation plus rigoureuse des penchants et des instincts de l'humanité ${ }^{26}$. La science de l'homme, la médecine, science du gouvernement ? Il est vrai que l'éphémère École Nationale d'Administration suscite la création d'un programme de cours d'anthropologie ${ }^{27}$. Il est incontestable aussi que les médecins sont bien en vue dans l'Assemblée constituante et qu'ils obtiennent avec le décret du 10 août le remplacement de l'ancien Conseil de santé par un Comité consultatif d’hygiène publique et, avec celui du 18 décembre 1848, le principe de la création d’un conseil d’hygiène dans chaque département. Mais l'accomplissement des mesures prônées par le corps médical fut souvent rejeté ou différé. A l'image de la présidence malheureuse de Buchez, l'illusion ne dura pas et les médecins reconnurent assez rapidement que la Seconde république n’avait pas établi une société conforme à leurs prescriptions.

Un sympathisant phrénologiste chercha à analyser l'échec de la Révolution de 1848. Dans un ouvrage publié en 1850 et prudemment dédicacé à Louis-Napoléon Bonaparte, Franklin-Berger explique que les révolutionnaires auraient dû s’appuyer sur la doctrine de Gall. La Constitution de la Seconde République ne permet pas le «bonheur public » car la population est formée d’individus trop différents ${ }^{28}$. Pour ce phrénologiste amateur, le bon législateur « physiologiste » doit tenir compte de la constitution organique de l'homme, composée de trois zones «molléculaires ». L’instinct, les sentiments et l'intelligence sont les trois « portes » par lesquels

\footnotetext{
25. J. Léonard, La médecine entre les savoirs et les pouvoirs, Paris, Aubier, 1981, pp. 219-224.

${ }^{26}$. J. Guérin, « La république et la médecine », Gazette médicale de Paris, 1848, 26 février-4 mars, n9-10, pp. 155-56.

27 . Le programme manuscrit de ce cours d' " économie de la population est conservé aux Archives nationales série F17 carton $\mathrm{n}^{\circ}$ 4142

${ }^{28}$. Victor Franklin-Berger, La révolution de Février et sa constitution, jugées par la phrénologie : de l'influence de cette doctrine sur l'économie sociale, Paris, Paul Dupont, 1850, pp. 23-26.
} 
le législateur peut agir sur les individus. Or l'histoire montre selon Franklin-Berger que la plupart des lois s’adressent aux instincts. Sparte a joué sur « les passions grossières » et les «appétits cyniques », tout comme le Coran de Mahomet, les Empires d'Orient et de Turquie. A Athènes même, la loi d'ostracisme était une reconnaissance implicite de l'organe phrénologique de l’amour de la localité, situé dans la zone instinctive. Même Rome fut entièrement régie par une législation qui s’appuyait sur les instincts et il fallut attendre le christianisme pour que les lois en appellent enfin à l'aire cérébrale des sentiments. Durant toute la période chrétienne, « les instincts et les sentiments sont en exercice, la raison souffre,... mais elle se tait ». Les coutumes expriment cette nouvelle conception du pouvoir en mobilisant essentiellement les sentiments d'amour-propre, d'orgueil, d'imitation, d'espérance et de merveilleux... ${ }^{29}$. Arguant du fait que les quatre sixièmes de l'humanité sont composés d'hommes sans éducation «à têtes aplaties et arrières », ne fonctionnant qu'à l'instinct, le phrénologiste propose d’instituer une éducation différentielle : il s’agirait d’établir dans des écoles de campagnes un enseignement simple en rapport avec la profession que l'individu peut exercer, tandis qu'un enseignement plus complet serait dispensé dans les villes. Quant aux génies, ils n’avaient pas besoin d’instituteurs... Ultime voix phrénologique sur la question scolaire, Franklin-Berger ne fut pas entendu et vingt ans après sa dernière réunion, le dernier président de la Société phrénologique reconnaissait que la théorie de Gall était totalement déconsidérée et « délaissée dans le mouvement social » ${ }^{30}$. Entretemps, la théorie des dégénérescences de B.-A. Morel avait séduit le champ anthropo-médical. Si elle n'inaugurait pas un changement radical dans la représentation de l'homme, elle marquait toutefois le succès d'une vision moins volontariste et un état des lieux pessimiste, accusant l'état de décadence de la société ${ }^{31}$.

\section{Un tournant dans les sciences de l'homme?}

«Le pessimisme a fait de grands progrès, explique Charles de Rémusat en 1860, et notre temps, qui a passé pour s'enorgueillir de ses oeuvres, compte aujourd'hui plus de censeurs que d'enthousiastes » ${ }^{32}$. Le constat vaut aussi après 1848 pour les sciences de l'homme. Cette évolution peut s’illustrer dans le champ scientifique à travers celle du terme de « dégénération ». Fréquent dans l'histoire naturelle du XVIIIe siècle, il exprime un potentiel positif de la nature humaine. L'espèce, une mais divisible, peut bien s’altérer ici en Nègres, là en Jaunes, ailleurs en Rouges, toutes ces variétés restent accessibles à une régénération, que peut apporter la religion

\footnotetext{
${ }^{29}$. Ibid., pp. 33-34.

30. Fossati, op. cit., 1869, p. 16.

${ }^{31}$. Sur cette transition en France, voir D. Pick, Faces of Degeneration. A European Disorder, c. 1848 - c. 1918, Cambridge, Cambridge University Press, 1989, pp. 37-73.

32. C. de Rémusat, « Du pessimisme politique », Revue des Deux-Mondes, 1860, vol. 38, p. 729.
} 
ou la civilisation occidentale. Cette acception tombe peu à peu en désuétude au XIXe siècle, avec quelques exceptions. L'anthropologie catholique affirme encore avec force l'unité morale et physique de l'espèce humaine. L'histoire du genre humain ? Elle est «consolante » juge Eusèbe de Salles en 1849 car elle « démontre combien la dégradation morale peut être empêchée par la continuité des efforts intellectuels !». La science de l’homme appelle en conséquence à la vigilance morale. "L'albinos et le crétin peuvent être considérés comme deux sentinelles placées en permanence aux deux extrémités de la famille humaine, explique Eusèbe de Salles : l'albinos console le nègre, en lui faisant entrevoir la régénération ; le crétin tempère l'orgueil des blancs par la menace de la décadence ! ${ }^{33}$.

Avec la montée de l'intérêt pour la question de l'hérédité, attestée par les thèses de médecine, le concours du prix Civrieux de 1843 et le succès du traité de Lucas paru en 1847, la connotation négative du terme de «dégénération » se renforce. Au lieu d’exprimer la plasticité de l'espèce sous forme d'accidents ostéologiques ou physiologiques réversibles, elle en signale désormais des altérations pathologiques ${ }^{34}$. Morel consacre cette tendance dans une théorie des dégénérescences qui met au premier plan l’hérédité (acquise) des phénomènes morbides. Ce n’est plus alors la mort des individus qui est en jeu, mais celle des races ${ }^{35}$. Sur ce point précis comme sur beaucoup d'autres, l'aliéniste porte une théorie charnière car son catholicisme social milite d’abord pour une régénération des peuples par la religion. L’illusion ne durera guère toutefois, et moins encore dans les faubourgs de métropole que pour les peuples colonisés. Les dégénérés de nos villes ? Ils figurent les crétins d’Eusèbe de Salles. Ils sont « la personnification des diverses dégénérescences dans l'espèce, et le mal qui les engendre constitue pour les sociétés modernes un danger plus grand que ne l'était pour les sociétés anciennes l'invasion des barbares ${ }^{36}$. Si Morel se défendit d'être fataliste, il se résigna vite. Loin de chercher comme les phrénologistes à «perfectionner la race », il veut seulement endiguer sa dégénérescence. Un signe parmi d’autres de son renoncement : l’aliéniste de Saint-Yon annonce en 1857 une suite à son Traité des dégénérescences. Il s’agit d’un Traité d'hygiène physique et morale permettant de «prévenir et de combattre efficacement les causes des dégénérescences de l’espèce humaine ».

\footnotetext{
${ }^{33}$. F. Eusèbe de Salles, Histoire générale des races humaines ou philosophie ethnographique, Paris, Benjamin Duprat, 1849, p. 299.

${ }^{34}$. Prosper Lucas, Traité philosophique et physiologique de l'hérédité naturelle, Paris, J.-B. Baillière, 1847, 2 vol. Sur l'origine et les variations sémantiques des termes "dégénération » et "dégénérescence » : Claude Bénichou, «Enquête et réflexions sur l’introduction des termes «dégénére(r), «dégénération », «dégénérescence » dans les dictionnaires et encyclopédies scientifiques françaises à partir du 17ème siècle ", Documents pour l'histoire du vocabulaire scientifique, 1983, n 5, pp. 1-62. ${ }^{35}$. C. Blanckaert, «L'ethnographie de la décadence. Culture morale et mort des races (XVIIe-XIXe siècles), Gradhiva, 1992, $\mathrm{n}^{\circ}$ 11, pp. 47-65.

36. Bénédict-Augustin Morel, Traité des dégénérescences physiques, intellectuelles et morales de l'espèce humaine et des causes qui produisent ces variétés maladives, Paris, Baillière, 1857, p. 660.
} 
L’ouvrage n'est jamais paru. L' « anthropologie morbide » est une anthropologie de l'échec. La nouvelle mission sociale de cette médecine n'est plus d'aller de l'avant mais d'éviter une nouvelle chute, de «régénérer le peuple » en combattant l'athéisme et le socialisme, affirme en 1858 le docteur Lepelletier de la Sarthe ${ }^{37}$. Pour que la société soit harmonique, chaque type social doit se contenter de cultiver la vertu qui lui est propre : le prêtre la pureté, le magistrat la justice, le militaire le courage, le savant la véracité, l'artiste la décence, l’industriel l'amour du travail, l’opulent la bienfaisance et le prolétaire...la «résignation » ${ }^{38}$. «Aujourd’hui, diagnostique Lepelletier, tous les travailleurs sont parfaitement libres : ils ne relèvent que de leur amour pour le travail, de leur aptitude et de leur volonté de l'exercer [...] S'ils veulent s'élever dans leur propre estime, dans celle de la société qui les encourage et les honore, il faut qu'ils emploient consciencieusement leur temps lorsqu'ils sont à la journée ; qu'ils s'appliquent toujours à la bonne confection des produits, qu'ils prennent une part sincère aux intérêts de leurs maîtres... » ${ }^{39}$. Il s'agit surtout de ne pas gaspiller sa force productive en se mêlant de faire de la politique. La devise du «système social » d'Almire Lepelletier est «Religion-famillepropriété ». La liberté d’un individu est de se mouvoir dans l’espace que lui assigne la société. Le peuple surtout doit se méfier des mirages. «Égalité! fraternité, voilà donc les deux expressions magiques avec lesquelles on a précipité les nations, en apparence les plus civilisées, dans les ruineux et sanglants désordres de l'insurrection, de la guerre civile, des interminables révolutions ${ }^{40}$. Cette voix qui émane de l'Académie de médecine, conforme en tout point à celle d'un Morel, est loin de faire l'unanimité dans la communauté médicale. Mais elle a les faveurs du nouveau régime. Et tout bon docteur ne sait-il pas que «les exercices et la fatigue musculaire diminuent et annulent les forces de l'intelligence » ${ }^{41}$

Quelques médecins ont tenté de relier les événements de 1848 avec la pathologie mentale. Un débat initié par le phrénologiste Jacques-Étienne Belhomme s’est ouvert dès 1848 à l’Académie de médecine pour apprécier l’influence des désordres politiques dans la production de la folie ${ }^{42}$. Cette question, qui n’est pas neuve, ne provoque nul consensus. Si l’on cherche aussi à relier sporadiquement contestation politique et pathologie individuelle, on est loin de voir dans la première la conséquence de la seconde. A cette époque, seuls des aliénistes comme Esquirol ou

\footnotetext{
37. Almire Lepelletier de la Sarthe, Illusions et réalités ou Régénération des peuples, Paris, Guillaumin et Cie, 1858.

${ }^{38}$. A. Lepelletier de la Sarthe, Du système social. Ses applications pratiques à l'individu, à la famille, à la société dans l'intérêt du bien-être, du bonheur et de la civilisation des peuples, Guillaumin et Cie, 1855, vol. I, pp. 72-94.

39. Ibid., vol. I, p. 313.

40. Ibid., vol. II, p. 669.

${ }^{41}$. Louis-Désiré Véron, Mémoires d’un bourgeois de Paris, Paris, Gabriel de Gonet, 1853, vol. I, p. 346.

42 . Jacques-Etienne Belhomme, «Influence des évènements et des commotions politiques sur le développement de l'aliénation mentale. Rapport », Bulletin de l’Académie de médecine, 1848, t. XIV, p. 592.
} 
Calmeil se sont directement intéressés aux mouvements collectifs «pathologiques » à travers le cas de la sorcellerie, interprétée comme une démonomanie. Un certain C. Th. Groddeck s'avisera pourtant de soutenir une thèse de médecine deux ans après 1848. Son titre est volontairement polémique : De la maladie démocratique. Nouvelle espèce de folie ${ }^{43}$. Groddeck affirme qu'un instinct d'imitation est à l'origine des phénomènes de contagion morale. Cet instinct peut être source de bien comme de mal, seule compte sa direction extérieure. Or celle-ci a pris un très mauvais tour en 1848 car l'idée de démocratie n’est que le produit d'une exaltation morbide combinée à un «amour de la liberté sans direction ni limite ». Le signe concret de la folie collective de 1848 résidait dans la volonté de limiter le pouvoir de l’État au bénéfice du peuple. «La révolution de Février avait renversé toutes les barrières de la compression, écrit Groddeck. Sortie du sang et des ruines des barricades, la souveraineté du peuple leva sa tête menaçante. A la nouvelle d'un événement qui devait imprimer au monde une secousse terrible, une excitation sourde mit en mouvement tous les esprits. Bientôt éclatèrent l'espoir, la crainte, la passion de la liberté, dans des pays que d'anciens événements ou la puissance de quelques génies révolutionnaires avaient préparé à la conquête de l'indépendance. Dans l'attente universelle, l'instinct d'imitation était un instrument tout prêt pour les agitateurs. Les oiseaux de la tempête prirent leur envol. L'étincelle partie de la France trouva par toute l'Europe des matières inflammables, et l'incendie fut allumé ${ }^{44}$. Quelle que soit la violence symbolique de cette interprétation, sa diffusion n’est pas comparable à la vague d’historiographie médicalisante des lendemains de la Commune de Paris. D’abord parce que l'argumentation de ce médecin est centrée sur le contexte politique outre-Rhin. Pour Groddeck, la thérapie réside dans l’unification rapide de tous les États allemands. Ensuite parce que cette thèse fut essentiellement commentée en France par l'aliéniste conservateur A. Brierre de Boismont, sans susciter de véritable débat ou de reprise.

C’est peut-être l'implication directe des médecins dans la révolution de 1848 qui explique leur relatif silence rétrospectif sur les événements politiques. Critiquer trop directement une révolution à laquelle ils avaient pris part, c’était reconnaître ostensiblement leur propre échec, discréditer leur prétention à produire une nouvelle forme de gouvernementalité. Les jugements furent donc indirects, et on peut en trouver les signes dans les inflexions globales du savoir scientifique. Le rôle essentiel de Buchez dans le succès de la théorie des dégénérescences est

\footnotetext{
${ }^{43}$. C.-Th. Groddeck, Die Demokratische Krankheit, eine neue Wanhsinns form ou De morbo democratico, nova insaniae forma, Berlin, 1850. Commentée par Brierre de Boismont dans les Annales médico-psychologiques, 1850, vol. II , pp. 519-523. Traduite en France par l'auteur et publiée la même année sous le titre De la maladie démocratique. Nouvelle espèce de folie, Paris, Germer Baillière, 1850.

44. Ibid., p. 51.
} 
bien connu, tout comme le virage conservateur d'Auguste Comte ${ }^{45}$. Le tournant qui s'amorce en ce milieu de siècle dans les sciences de l’homme n’est pas spécifique à Morel. 1848 marque ainsi peut-être la fin d’une période commencée sous la révolution de 1789 avec la génération des Idéologues. Période durant laquelle le rapport de la science au politique a pu se penser sur le mode de la subordination de la seconde à la première. Les phrénologistes voulaient tout réformer à l'aune de leur théorie : sculpture, dessin, éducation, système judiciaire, traitement des aliénés etc. C’est ce messianisme scientifique qui a été sacrifié par les savants sur l'autel de la révolution. Le scientisme d'un Renan n’est plus d'actualité ${ }^{46}$. Au delà de la seule phrénologie, 1848 marque la fin d'une certaine conception de la science caméraliste, autonome et souveraine, qui dicte ses principes à un pouvoir politique qui n'a plus qu'à appliquer ses sentences. Ce qui se termine alors, ce n'est pas tant l'influence des discours scientifiques sur le politique que le projet de révolution sociale déduit des connaissances scientifiques. La première moitié du XIXe siècle avait été marquée par la multiplication d’utopies scientifiques au service du politique. Ce que la révolution de 1848 sanctionne d’une certaine manière, c'est l'échec ou - pour éviter un jugement normatif - le refus d’appliquer directement ces visions «révolutionnaires » dans l'art du gouvernement. Après 1848, il n’y aura plus de théorie comparable à la phrénologie cherchant ouvertement à réformer toute la société et à l’organiser selon ses principes. Même le très militant Beunaiche de Lacorbière commencera en 1853 un nouvel ouvrage sur la phrénologie par une mise en garde contre les « utopies $»{ }^{47} \ldots$

Hypothèses bien sûr, d'autant plus fragiles et provisoires qu'elles englobent des sciences de l'homme en cours de constitution. Hypothèses qui devraient surtout tenir compte des transitions chronologiques, qui ne se recoupent que partiellement entre les différentes sciences. Ce tournant du milieu de siècle demande à être testé sur plusieurs disciplines en voie d'institutionnalisation. Un changement incontestable est perceptible dans le champ de la médecine mentale, où la doctrine des dégénérescences de Morel apparaît comme une réponse quasi providentielle aux apories de la doctrine de l'aliénation mentale, qui a connu un échec important en matière d’expertise médico-légale. On peut aussi percevoir ce même mouvement en ethnologie, où les prétentions hégémoniques de la phrénologie sont rapidement concurrencées dès les années 1840 par une raciologie différentialiste à laquelle elle sacrifiera sans pouvoir s’y adapter vraiment ${ }^{48}$.

\footnotetext{
45. Sur le rôle de Buchez dans le champ psychiatrique, voir Ian Dowbiggin, La folie héréditaire, Paris, E.P.E.L., 1993.

46 . Ernest Renan, L’avenir de la science, Paris, Flammarion, 1995 (1ère édition 1890, rédigé en 1848).

47. J.-B. Beunaiche de La Corbière, De l'influence que doit exercer la phrénologie sur les progrès ultérieurs de la philosophie et de la morale, Paris, Victor Masson, 1853, pp. 29-30.

48 . Les tensions entre les deux anthropologies furent très fortes par exemple lors de la seconde circumnavigation de Dumont d’Urville. M. Renneville, «Un terrain phrénologique dans le Grand Océan (autour du voyage de Dumoutier à bord de
} 
Ce n'est pas que ces nouvelles doctrines refusent de s’immiscer dans le politique. Mais la stratégie a changé : loin de s’essayer à renverser les institutions, les discours anthropologiques obtiendront d'autant plus de succès qu'ils conforteront la hiérarchisation et le classement des sujets au fondement de nos démocraties représentatives.

\section{Institutions, mythes et histoire(s)}

En 1836-37, la revue des phrénologistes portait en sous titre : «Journal des applications de la physiologie animale à la physiologie sociale ». Un énoncé étrange en premier lecture mais qui rappelle combien le mouvement phrénologique s’inscrivait dans une vague de pensée beaucoup plus large. Le premier XIXe siècle était à la recherche d'une harmonie perdue. Entre les nostalgiques de l'ordre ancien et ceux qui affirmaient la nécessité d'organiser rationnellement et scientifiquement un nouvel ordre social, combien d'hybrides, d'hésitants, d'éclectiques, de catéchismes matérialistes et de bibles nouvelles? Tous ces théoriciens s'accordaient implicitement sur un point : l'image juridique du contrat était insuffisante pour justifier le lien social. Les métaphores organicistes, assimilant le corps social à un corps individuel, furent l'une des réponses à cette lacune. De Saint-Simon à Durkheim en passant par les phrénologistes, Herbert Spencer, René Wörms ou Alfred Espinas, les sciences de l’homme apportèrent ici leur caution à la légitimation de l'ordre présent ${ }^{49}$. Plus que des figures de style, les analogies naturalistes furent la grammaire de nouveaux mythes. L’histoire croisée des sciences et de la politique gagnerait sur cet épisode à dialoguer avec l'anthropologie. Mary Douglas proposait par exemple il y a quelques années une sociologie de la connaissance qui s’appliquait aussi bien aux représentations des savoirs populaires qu'aux discours savants ${ }^{50}$. Reprenant un programme énoncé par E. Durkheim et L. Fleck, son étude comparée de quelques systèmes de classification visait à démontrer qu’une institution n’est légitimée que lorsqu’une convention cognitive la fonde en nature ${ }^{51}$. L'anthropologue ajoutait que cette relation ne pouvait acquérir sa fonction de légitimation que si l'indépendance des champs mis en relation était posée. Toute institution serait ainsi productrice de mythes qui ne nient pas le politique mais l'inscrive dans des rapports d'analogie avec ce qu'il n'est pas: physiologie animale, anatomie comparée, pathologie mentale, psychologie des peuples etc. Ce mode de raisonnement analogique - dont on reconnaît

L'Astrolabe en 1837-40)" in C. Blanckaert (Ed.), Le terrain des sciences humaines (Instructions et enquêtes. XVIIIe-XXe siècle), Paris, L'Harmattan, 1996, pp. 89-138.

${ }^{49}$. Sur la résurgence de l’organicisme après la Révolution française, voir Frank E. Manuel, «From Equality to Organicism » in Maryanne Cline Horowitz (Ed.), Race, Class and Gender in Nineteenth-Century Culture, New York, University of Rochester Press, 1991, pp. 3-18. Pour une analyse épistémologique des métaphores scientifico-politique dans le XIXe français : Judith Schlanger, Les métaphores de l’organisme, Paris, L’Harmattan, 1995 (1971).

${ }^{50}$. M. Douglas, De la souillure (Etudes sur la notion de pollution et de tabou), Paris, La Découverte, 1992. 
facilement l'existence chez les «peuples primitifs » - fut constamment mis en oeuvre au XIXe siècle, tant en science qu'en politique. Or c’est précisément durant cette période que le positivisme a imposé une représentation de la production des connaissances scientifiques indépendante du politique. La science doit être l'interprète de la nature pour la politique, jamais l’inverse. Ce fut longtemps l'arme rhétorique absolue. Lorsque Friedrich Tiedemann juge que l'esclavage n'est pas une institution de nature, les savants français passent outre le décret d'abolition du 27 avril 1848 et dénoncent sous le Second Empire la non-scientificité d’un tel jugement pour l' « intention politique » qu'il cache... ${ }^{52}$. Dans cette religion scientiste, un tabou frappe toute théorie dont on reconnaît l'origine politique. Le plus étonnant peut-être, c'est que l'histoire des sciences a elle-même longtemps reproduit ce genre historiographique, qui juge une science impure ou souillée dès qu'un contact avec le politique peut être établi. A l'inverse, l'anthropologie de Douglas donne des clefs pour repenser cet ancien régime épistémologique et analyser de front la contribution des représentations scientifiques et politiques à la formation du lien social. D’autres auteurs pourraient certainement être convoqués. Mais est-on bien sûr que la tribu des historiens ait fait, sur cette question, sa révolution?

Marc Renneville

\footnotetext{
${ }^{51}$. M. Douglas, Ainsi pensent les institutions, Paris, Usher, 1989.

${ }^{52}$ C. Blanckaert, «La science de l'homme entre humanité et inhumanité » in Des sciences contre l'homme, Paris, Autrement, 1993, vol. I, p. 19.
} 